\title{
Tarifbindung und betriebliche Interes- senvertretung: Ergebnisse aus dem IAB-Betriebspanel 2013
}

\author{
Der Beitrag präsentiert aktuelle Ergebnisse zur Tarifbindung und der betrieblichen Inte- \\ ressenvertretung. Er knüpft damit an die alljährlich in den WSI-Mitteilungen publizierte \\ Berichterstattung des Instituts für Arbeitsmarkt- und Berufsforschung (IAB) zur Reich- \\ weite von Tarifbindung und Mitbestimmung an. Die Befunde basieren auf Daten des \\ IAB-Betriebspanels, das als einzige repräsentative Datenquelle entsprechende Auskünfte \\ in einer langen Zeitreihe für die Gesamtwirtschaft liefert.
}

PETER ELLGUTH, SUSANNE KOHAUT

\section{Einleitung}

Das deutsche System der Arbeitsbeziehungen wird durch die gesetzlichen Bestimmungen zur Tarifautonomie und zur betrieblichen Interessenvertretung durch Betriebsräte getragen. Überbetriebliche Branchen- oder Flächentarifverträge spielen eine wesentliche Rolle bei der Regelung von Arbeitsbedingungen und bei der Lohnfindung. Sie werden meist für Regionen und Branchen ausgehandelt und sorgen dort für einheitliche Wettbewerbsbedingungen bei den Arbeitskosten. Während der Laufzeit der Verträge herrscht Betriebsfrieden. Der mit Tarifverhandlungen verbundene Aufwand liegt bei den Verbänden, was die Betriebe entlastet.

Löhne und Arbeitsbedingungen können jedoch nicht nur auf Branchenebene über (Flächen)Tarifverträge, sondern auch auf Betriebs- oder Unternehmensebene (Firmentarifverträge) oder in individuellen Arbeitsverträgen geregelt werden. Letzteres gilt, soweit keine Tarifbindung besteht oder die individuell ausgehandelten Konditionen günstiger als die tariflichen sind. Individuelle Arbeitsverträge werden vor allem in kleineren, nicht tarifgebundenen Betrieben geschlossen. Für größere Firmen wird der Verwaltungsaufwand schnell zu groß, wenn mit jedem Beschäftigten einzeln ein Arbeitsvertrag verhandelt werden muss. Für solche Betriebe sind deshalb Firmentarifverträge eine interessante Alternative.

Im Arbeitsrecht haben Tarifverträge Vorrang gegenüber Betriebsvereinbarungen und Einzelarbeitsverträgen, was vor allem meint, dass nicht zuungunsten der Beschäftigten von tariflichen Regelungen abgewichen werden kann. Insoweit können sie auch als Mindestarbeitsbedingungen interpretiert werden. Ihren Kritikern gelten Branchentarifverträge häufig als zu starr, da sie branchenweit zwischen Gewerkschaften und Arbeitgeberverbänden ausgehandelt werden und somit nicht die Situation jedes einzelnen Betriebs berücksichtigen können. Mittlerweile sind jedoch zwischen den Tarifvertragsparteien vereinbarte Öffnungsklauseln in Branchentarifverträgen weit verbreitet, die explizit betriebliche Gestaltungsmöglichkeiten zur Unterschreitung tariflicher Standards anbieten. Damit haben betriebsbezogene Regelungen und die betrieblichen Interessenvertretungen an Bedeutung gewonnen.

Eine weitere Form der Differenzierung, die allerdings eher selten diskutiert wird, besteht für Betriebe in der übertariflichen Entlohnung. Hierbei erhält meist nur ein Teil der Mitarbeiter höhere Effektivlöhne als in den Tarifverträgen vereinbart.

Der vermuteten „Erosion des Flächentarifvertrags“ und dem Thema „Stärkung der betrieblichen Aushandlungsebene“ wird in der Öffentlichkeit unverändert große Aufmerksamkeit entgegengebracht. Weniger im Fokus stehen die Betriebe, die formal zwar nicht tarifgebunden sind, sich jedoch freiwillig an einem Branchentarifvertrag orientieren. Um die tatsächliche Bedeutung der Flächentarifverträge für die Wirtschaft abschätzen zu können, müssen auch diese Betriebe betrachtet werden. 
TABELLE 1

\section{Beschäftigte in tarifgebundenen Betrieben 2013 nach Wirtschaftszweig}

Anteile in Prozent

\begin{tabular}{l|c|c|}
\hline & \multicolumn{2}{c}{$\begin{array}{c}\text { Branchen- } \\
\text { tarifvertrag }\end{array}$} \\
\hline Landwirtschaft u.a. & West & Ost \\
\hline Energie/Wasser/Abfall u. Bergbau & 50 & 14 \\
\hline Verarbeitendes Gewerbe & 75 & 42 \\
\hline Baugewerbe & 55 & 20 \\
\hline Großhandel, KfZ-Handel und -reparatur & 69 & 51 \\
\hline Einzelhandel & 37 & 17 \\
\hline Verkehr u. Lagerei & 41 & 27 \\
\hline Information u. Kommunikation & 42 & 20 \\
\hline Finanz- und Versicherungsdienstleistungen & 15 & $(7)$ \\
\hline Gastgewerbe u. Sonst. Dienstleistungen (DL) & 81 & 57 \\
\hline Gesundheit u. Erziehung/Unterricht & 53 & 28 \\
\hline Wirtschaftl., wissenschaftl. u. freiberufl. DL & 52 & 83 \\
\hline Org. ohne Erwerbscharakter & 54 & 30 \\
\hline Öffentl. Verwaltung/ Sozialversicherung & 43 & 45 \\
\hline Gesamt & 53 & 26 \\
\hline
\end{tabular}

\begin{tabular}{|c|c|}
\hline $\begin{array}{c}\text { Haus-/Firmen- } \\
\text { tarifvertrag }\end{array}$ \\
\hline West & Ost \\
\hline$(2)$ & $(5)$ \\
\hline 15 & 29 \\
\hline 12 & 14 \\
\hline 2 & $(6)$ \\
\hline 7 & 12 \\
\hline 2 & 6 \\
\hline 13 & 12 \\
\hline$(19)$ & 16 \\
\hline 2 & $(0)$ \\
\hline 3 & 6 \\
\hline 10 & 23 \\
\hline 4 & 7 \\
\hline 12 & 13 \\
\hline 9 & 16 \\
\hline 8 & 12 \\
\hline
\end{tabular}

\begin{tabular}{|c|c|c|c|}
\hline \multicolumn{4}{|c}{ Kein Tarifvertrag } \\
\hline West & $\begin{array}{c}\text { davon Orientierung } \\
\text { am Branchentarif }\end{array}$ & Ost & $\begin{array}{c}\text { davon Orientierung } \\
\text { am Branchentarif }\end{array}$ \\
\hline 48 & 47 & 81 & 51 \\
\hline 10 & 48 & 29 & 42 \\
\hline 33 & 62 & 66 & 51 \\
\hline 29 & 65 & 43 & 65 \\
\hline 56 & 58 & 72 & 49 \\
\hline 57 & 65 & 67 & 52 \\
\hline 45 & 39 & 68 & 45 \\
\hline 65 & 32 & 78 & 26 \\
\hline 16 & 39 & 43 & 30 \\
\hline 57 & 50 & 68 & 45 \\
\hline 37 & 65 & 47 & 53 \\
\hline 53 & 37 & 49 & 36 \\
\hline 35 & 61 & 59 & 54 \\
\hline 2 & 63 & 2 & $(81)$ \\
\hline 40 & 53 & 53 & 48 \\
\hline & & & \\
\hline
\end{tabular}

Werte in Klammern aufgrund geringer Fallzahl wenig belastbar.

Quelle: IAB-Betriebspanel (2013).

Auf betrieblicher Ebene regelt das Betriebsverfassungsgesetz (BetrVG) die institutionalisierte Interessenvertretung der Beschäftigten. Formal herrscht eine klare funktionale Trennung zwischen der Mitbestimmung auf Branchenebene und auf betrieblicher Ebene. Die Praxis jedoch ist geprägt von einem vielfältigen Ineinandergreifen beider Ebenen mit wechselseitigen Abhängigkeiten. Betriebsräten obliegt die Umsetzung und Überwachung geltender Tarifverträge.

Seit 1996 werden sowohl für West- als auch für Ostdeutschland jährlich Informationen zur Tarifbindung und zur Existenz eines Betriebsrats erhoben. 2013 wurden zudem die tarifgebundenen Betriebe genauer befragt, ob sie übertariflich entlohnen, wie viele Mitarbeiter in den Genuss dieser Leistungen kommen und in welcher Höhe diese $\mathrm{Zu}$ lagen gewährt werden. Die aktuellen Ergebnisse beruhen auf Angaben von rund 15.700 Betrieben in beiden Landesteilen. Aufgrund des Aufbaus der Zufallsstichprobe sind die Ergebnisse repräsentativ für die rund 2,1 Mio. Betriebe mit mindestens einem sozialversicherungspflichtig Beschäftigten. Insgesamt sind in diesen Betrieben etwa 36,7 Mio. Personen beschäftigt. ${ }^{1}$

\section{Ausmaß und Entwicklung der Tarifbindung}

In der folgenden Darstellung wird zwischen der Tarifbindung der Betriebe und der Beschäftigten unterschieden. Betrachtet man zunächst die Beschäftigten, so arbeiten im Jahr 2013 hochgerechnet rund $52 \%$ der westdeutschen und etwa $35 \%$ der ostdeutschen Beschäftigten in einem Betrieb, der einem Branchentarifvertrag unterliegt (Tabelle 1). Firmentarifverträge gelten für $8 \%$ der westdeutschen und $12 \%$ der ostdeutschen Beschäftigten. Für rund $40 \%$ der westdeutschen und $53 \%$ der ostdeutschen Arbeitnehmer gibt es keinen Tarifvertrag. Jeweils rund die Hälfte dieser Arbeitnehmer profitiert jedoch indirekt von Tarifverträgen, da sich ihre Betriebe nach eigenen Angaben daran orientieren. Das Ausmaß der Tarifbindung va-

1 Für weitere Informationen zum IAB-Betriebspanel siehe Ellguth et al. (2014). Die Erhebung wird von TNS Infratest Sozialforschung durchgeführt. 
TABELLE 2

Tarifbindung der Betriebe 2013 nach Wirtschaftszweig

Angaben in Prozent

\begin{tabular}{l|c|c}
\hline & \multicolumn{2}{|c}{$\begin{array}{c}\text { Branchen- } \\
\text { tarifvertrag }\end{array}$} \\
\hline Landwirtschaft u.a. & West & Ost \\
\hline Energie/Wasser/Abfall u. Bergbau & 34 & 6 \\
\hline Verarbeitendes Gewerbe & 62 & 29 \\
\hline Baugewerbe & 30 & 12 \\
\hline Großhandel, KfZ-Handel und -reparatur & 54 & 41 \\
\hline Einzelhandel & 24 & 9 \\
\hline Verkehr u. Lagerei & 30 & 13 \\
\hline Information u. Kommunikation & 27 & 8 \\
\hline Finanz- und Versicherungsdienstleistungen & 4 & $(1)$ \\
\hline Gastgewerbe u. Sonst. DL & 40 & 15 \\
\hline Gesundheit u. Erziehung/Unterricht & 39 & 17 \\
\hline Wirtschaftl., wissenschaftl. u. freiberufl. DL & 36 & 12 \\
\hline Org. ohne Erwerbscharakter & 35 & 15 \\
\hline Öffentl. Verwaltung/ Sozialversicherung & 16 \\
\hline Gesamt & 30 & 13 \\
\hline & 34 & 94 \\
\hline
\end{tabular}

\begin{tabular}{|c|c|}
\hline $\begin{array}{c}\text { Haus-/Firmen- } \\
\text { tarifvertrag }\end{array}$ \\
\hline West & Ost \\
\hline$(1)$ & $(2)$ \\
\hline 6 & 9 \\
\hline 3 & 5 \\
\hline 1 & $(0)$ \\
\hline 2 & 2 \\
\hline 1 & 4 \\
\hline 4 & 6 \\
\hline$(4)$ & 3 \\
\hline 0 & $(0)$ \\
\hline 1 & 2 \\
\hline 3 & 7 \\
\hline 1 & 1 \\
\hline 15 & 11 \\
\hline 10 & 4 \\
\hline 2 & 3 \\
\hline & \\
\hline
\end{tabular}

\begin{tabular}{|c|c|c|c|}
\hline \multicolumn{4}{|c}{ Kein Tarifvertrag } \\
\hline West & $\begin{array}{c}\text { davon Orientierung } \\
\text { am Branchentarif }\end{array}$ & Ost & $\begin{array}{c}\text { davon Orientierung } \\
\text { am Branchentarif }\end{array}$ \\
\hline 65 & 45 & 92 & 47 \\
\hline 32 & 43 & 62 & 33 \\
\hline 67 & 54 & 83 & 48 \\
\hline 45 & 55 & 59 & 59 \\
\hline 73 & 48 & 89 & 43 \\
\hline 69 & 51 & 83 & 43 \\
\hline 70 & 27 & 86 & 40 \\
\hline 92 & 26 & 96 & 20 \\
\hline 60 & 33 & 85 & 9 \\
\hline 74 & 37 & 86 & 35 \\
\hline 62 & 58 & 78 & 53 \\
\hline 82 & 33 & 86 & 36 \\
\hline 46 & 59 & 72 & 44 \\
\hline 5 & 61 & 2 & 1951 \\
\hline 67 & 44 & 80 & 42 \\
\hline & & & \\
\hline
\end{tabular}

Werte in Klammern aufgrund geringer Fallzahl wenig belastbar.

Quelle: IAB-Betriebspanel (2013).

riiert zwischen den Wirtschaftszweigen stark, wobei sich die Bedeutung für die einzelnen Branchen von Jahr zu Jahr wenig ändert. Seit Jahren liegt die Tarifbindung bei den Finanz- und Versicherungsdienstleistungen und im Baugewerbe in beiden Landesteilen weit über dem Durchschnitt, während vor allem im Bereich der Information und Kommunikation Branchentarifverträge kaum eine Rolle spielen.

Aufgrund der starken Größenabhängigkeit der Tarifbindung ergibt sich auf Ebene der Betriebe, dass hochgerechnet rund $30 \%$ der westdeutschen, aber nur $17 \%$ der ostdeutschen Betriebe durch Branchentarifverträge gebunden sind. Haus- oder Firmentarifverträge gelten für $2 \%$ der Betriebe in den alten und etwa $3 \%$ der Betriebe in den neuen Bundesländern (Tabelle 2). Der Rest, also etwa $67 \%$ der westdeutschen und etwa $80 \%$ der ostdeutschen Betriebe, ist nicht tarifgebunden. Allerdings geben etwa $44 \%$ der nicht tarifgebundenen Betriebe in Westdeutschland und $42 \%$ in Ostdeutschland an, sich in ihren Einzelarbeitsverträgen an bestehenden Branchentarifen zu orientieren

Betrachtet man die Tarifbindung der Betriebe nach ihrer Größe (Tabelle 3), so zeigt sich wie in den Vorjahren, dass der Deckungsgrad mit zunehmender Beschäftigtenzahl steigt. Das gilt sowohl für die alten als auch für die neuen Bundesländer, wenn auch auf unterschiedlichem Niveau. Während für die Kleinbetriebe die Branchentarifverträge eine untergeordnete Rolle spielen, sind Großbetriebe mit über 500 Beschäftigten in der Mehrheit tarifgebunden. Ebenso nimmt die Bedeutung der Haus- bzw. Firmentarifverträge mit steigender Betriebsgröße zu. Insbesondere in ostdeutschen Großbetrieben mit 500 und mehr Beschäftigten spielen Firmentarifverträge eine große Rolle.

Seit Beginn der Erhebung 1996 bis Mitte der 2000er Jahre zeigt die Branchentarifbindung in den alten wie in den neuen Bundesländern eine stark rückläufige Tendenz. In Westdeutschland folgt danach zunächst eine Phase der Stabilisierung bis 2010, während in Ostdeutschland die Reichweite der Tarifbindung stetig - wenn auch nur in sehr kleinen Schritten - abnimmt. Auch in Westdeutschland ist nach einer „ruhigen“ Phase erneut ein Rückgang $\mathrm{zu}$ verzeichnen. $\mathrm{Ob}$ sich dieser Abwärtstrend fortsetzt werden die nächsten Jahre zeigen. In Abbildung 1 sind sowohl die gesamtwirtschaftliche Entwicklung als auch die 
Tarifbindung der Betriebe 2013 nach Betriebsgröße

Anteile in Prozent

\begin{tabular}{l|c|c}
\hline & \multicolumn{2}{c}{$\begin{array}{c}\text { Branchen- } \\
\text { tarifvertrag }\end{array}$} \\
\hline West & Ost \\
\hline 1 bis 9 Beschäftigte & 25 & 13 \\
\hline 10 bis 49 Beschäftigte & 41 & 26 \\
\hline 50 bis 199 Beschäftigte & 50 & 43 \\
\hline 200 bis 499 Beschäftigte & 61 & 50 \\
\hline 500 und mehr Beschäftigte & 76 & 54 \\
\hline Gesamt & $\mathbf{3 0}$ & $\mathbf{1 7}$ \\
\hline
\end{tabular}

\begin{tabular}{|c|c|}
\hline $\begin{array}{c}\text { Haus-/Firmen- } \\
\text { tarifvertrag }\end{array}$ \\
\hline West & Ost \\
\hline 1 & 2 \\
\hline 4 & 6 \\
\hline 9 & 13 \\
\hline 12 & 18 \\
\hline 12 & 28 \\
\hline 2 & 3 \\
\hline
\end{tabular}

Quelle: IAB-Betriebspanel (2013)

\begin{tabular}{|c|c|c|c|}
\hline \multicolumn{4}{|c|}{ Kein Tarifvertrag } \\
\hline West & $\begin{array}{c}\text { davon Orientierung } \\
\text { am Branchentarif }\end{array}$ & Ost & $\begin{array}{c}\text { davon Orientierung } \\
\text { am Branchentarif }\end{array}$ \\
\hline 74 & 40 & 86 & 40 \\
\hline 57 & 56 & 69 & 48 \\
\hline 42 & 59 & 44 & 53 \\
\hline 27 & 55 & 32 & 54 \\
\hline 12 & 59 & 19 & 48 \\
\hline 67 & 44 & 80 & 42 \\
\hline
\end{tabular}

Mitteilungen
Entwicklung der Branchentarifbindung in der Privatwirtschaft dargestellt. Betrachtet man zunächst die Gesamtwirtschaft in Westdeutschland, so ging die Flächentarifbindung bezogen auf die Beschäftigten von 1996 bis 2013 um 17 Prozentpunkte zurück. In Ostdeutschland belief sich dieser Rückgang auf 20 Prozentpunkte. Nahezu die gleiche Entwicklung ist zu beobachten, wenn nur die Privatwirtschaft betrachtet wird. Das bedeutet, dass der Rückgang der Branchentarifbindung durch die Betriebe der Privatwirtschaft getrieben wird, während die Flächentarifbindung im öffentlichen Sektor im betrachten Zeitraum weitgehend stabil blieb.

\section{Verbreitung und Ausmaß der über- tariflichen Entlohnung}

Angesichts des Mindestlohncharakters und der relativ geringen Differenzierung der Tariflöhne spielt die übertarifliche Entlohnung eine wichtige Rolle für die Ausgestaltung der effektiven Entgelte innerhalb der Betriebe, um beispielsweise besonders qualifizierte Beschäftigte zu halten oder die eigene Attraktivität als Arbeitgeber zu erhöhen. Übertarifliche Entlohnung erhöht zudem die Flexibilität der Betriebe, da bei veränderten wirtschaftlichen Rahmenbedingungen die übertariflichen Lohnbestandteile abgeschmolzen oder mit tariflichen Lohnerhöhungen verrechnet werden können. Der Anteil der Betriebe, die übertariflich entlohnen, sowie die Höhe der Lohnspanne geben Aufschluss über die relative Bedeutung der übertariflichen Entlohnung für das Lohnfindungssystem und die betrieblichen Gestaltungsspielräume. Bei der Entschei- dung, ob übertariflich entlohnt wird, auf welchen Personenkreis sich die zusätzlichen Leistungen erstrecken und welchen Umfang die Leistungen haben, hat der Betriebsrat allerdings kein Mitbestimmungsrecht.

Informationen über Verbreitung und Ausmaß der übertariflichen Entlohnung werden jährlich im IAB-Betriebspanel erhoben. 2013 wurde darüber hinaus erstmals der Anteil der Beschäftigten, die in den Genuss dieser höheren Effektivlöhne kommen, erfragt. 39 \% der westdeutschen und $24 \%$ der ostdeutschen tarifgebundenen Betriebe geben 2013 an, dass sie höhere Löhne als im

ABB.

Flächentarifbindung der Beschäftigten 1996-2013

Angaben in Prozent

- Gesamtwirtschaft West Gesamtwirtschaft Ost

- Privatwirtschaft* West $\diamond$ Privatwirtschaft* Ost

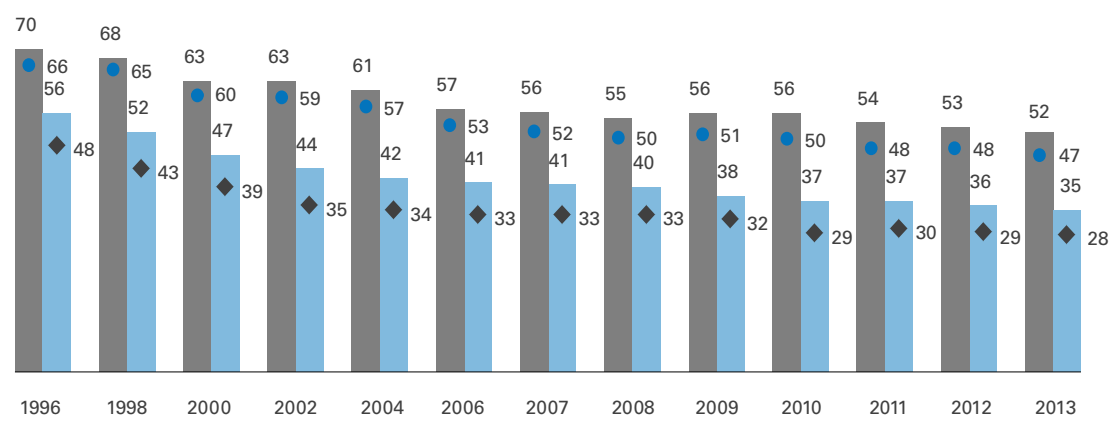

*ohne Landwirtschaft und Organisationen ohne Erwerbszweck.

Anmerkung: Seit 2010 verändertes Datenprüfverfahren, siehe Ellguth/Kohaut (2011, S. 243).

Quelle: IAB-Betriebspanel; Darstellung der Autoren. 
TABELLE 4

Übertarifliche Entlohnung tarifgebundener Betriebe 2013 nach Betriebsgröße*

Anteile in Prozent

\begin{tabular}{|c|c|c|c|c|c|c|}
\hline & \multicolumn{2}{|c|}{$\begin{array}{c}\text { Anteil übertariflich } \\
\text { entlohnender Betriebe }\end{array}$} & \multicolumn{2}{|c|}{$\begin{array}{l}\text { Anteil der begünstigten } \\
\text { Beschäftigten in den } \\
\text { betreffenden Betrieben }\end{array}$} & \multicolumn{2}{|c|}{$\begin{array}{l}\text { Höhe der übertariflichen } \\
\text { Entlohnung für } \\
\text { begünstigte Beschäftigte }\end{array}$} \\
\hline & West & Ost & West & Ost & West & Ost \\
\hline 1 bis 9 Beschäftigte & 30 & 19 & 71 & 67 & 11 & 12 \\
\hline 10 bis 49 Beschäftigte & 51 & 29 & 61 & 53 & 10 & 11 \\
\hline 50 bis 199 Beschäftigte & 50 & 31 & 50 & 52 & 10 & 10 \\
\hline 200 bis 499 Beschäftigte & 50 & 35 & 44 & 45 & 11 & 12 \\
\hline 500 und mehr Beschäftigte & 48 & 17 & 47 & 12 & 10 & 9 \\
\hline Gesamt & 39 & 24 & 64 & 59 & 10 & 11 \\
\hline
\end{tabular}

* Basis: privatwirtschaftliche Betriebe ohne Landwirtschaft und Organisationen ohne Erwerbszweck.

Quelle: IAB-Betriebspanel (2013).

Tarifvertrag vorgesehen zahlen. Jeweils $64 \%$ (West) bzw. 59 \% (Ost) der Beschäftigten profitieren von der höheren Bezahlung. Die Lohnspanne (bei den Beschäftigten, die in den Genuss der zusätzlichen Leistungen kommen) liegt in beiden Landesteilen bei rund $10 \%$.

Eine Betrachtung der übertariflichen Entlohnung nach Betriebsgröße (Tabelle 4) ergibt kein einheitliches Bild. Sowohl in West- als auch in Ostdeutschland scheinen vor allem die kleineren und mittelgroßen Betriebe mit zehn bis unter 500 Beschäftigten überdurchschnittlich häufig das Instrument der übertariflichen Entlohnung zu nutzen. Der Anteil der Beschäftigten, die mehr als den Tariflohn bekommen, nimmt tendenziell mit der Betriebsgröße ab, während die Höhe der Lohnspanne kaum Streuung über die Betriebsgrößenklassen hinweg aufweist.

Die Darstellung der übertariflichen Entlohnung nach Wirtschaftszweigen zeigt, dass im Bereich des Großhandels in beiden Landesteilen überdurchschnittlich häufig tarifgebundene Betriebe nach oben von den Löhnen abweichen, wobei der Anteil der Beschäftigten in Ost und West, die von den höheren Löhnen profitieren, eher im Durchschnitt liegt und auch die Höhe der Lohnspanne durchschnittlich bleibt. Besonders selten zahlen Betriebe im Bergbau und Energiebereich übertariflich. Auch das ist in West- wie Ostdeutschland zu beobachten. Insgesamt kann zwar eine deutliche Variation des Anteils übertariflich entlohnender Betriebe und des Anteils der Beschäftigten, die hiervon profitieren, festgestellt werden. Die Höhe der Lohnspanne variiert im Vergleich hierzu jedoch nur wenig (Tabelle 5).

\section{Verbreitung betrieblicher Interessen- vertretungen}

Neben der Tarifautonomie bildet die betriebliche Mitbestimmung die zweite tragende Säule im bundesdeutschen System industrieller Beziehungen. In den bisherigen Veröffentlichungen zur quantitativen Entwicklung der industriellen Beziehungen in Deutschland auf Basis des IABBetriebspanels war die betriebliche im Gegensatz zur sektoralen Ebene über Jahre als Hort weitgehender Stabilität und Kontinuität mit keinen bzw. geringen (inhaltlich nicht interpretierbaren) jährlichen Veränderungen beschrieben worden (siehe z. B. Ellguth 2007). Zuletzt war aber auch hier ein Abwärtstrend beim Anteil der Beschäftigten, deren Interessen durch einen Betriebsrat vertreten werden, konstatiert worden (vgl. Ellguth/Kohaut 2012). Vor diesem Hintergrund soll nun neben der Präsentation aktueller Strukturdaten besonderer Wert auf eine differenzierte Betrachtung der mittlerweile 18 (Ost) bzw. 21 (West) Jahre zurückreichenden Zeitreihe gelegt werden.

In Tabelle 6 sind die entsprechenden Daten zur Verbreitung von Betriebsräten in der Privatwirtschaft (Betriebe ab fünf Beschäftigte) $)^{2}$ für Westdeutschland seit 1993

2 Die Betrachtung der Betriebe ab fünf Beschäftigte ergibt sich aufgrund der Gesetzeslage, die erst ab dieser Betriebsgröße die Wahl eines Betriebsrats zulässt. Damit sind immerhin rund $39 \%$ der privatwirtschaftlichen Betriebe im Westen und $46 \%$ im Osten per se ohne eine gesetzlich legitimierte Interessenvertretung. Allerdings stellt sich die Situation weniger dramatisch dar, wenn wir von der Betriebs- zur Beschäftigtenperspektive wechseln und die entsprechenden Anteilswerte betrachten. Da in der großen 


\section{Übertarifliche Entlohnung tarifgebundener Betriebe 2013 nach Wirtschaftszweig*}

\begin{tabular}{l|c|c}
\hline & $\begin{array}{c}\text { Anteil übertariflich } \\
\text { entlohnender } \\
\text { Betriebe }\end{array}$ \\
\hline Energie/Wasser/Abfall u. Bergbau & West & Ost \\
\hline Verarbeitendes Gewerbe & 26 & 18 \\
\hline Baugewerbe & 43 & 26 \\
\hline Großhandel, KfZ-Handel und -reparatur & 36 & 28 \\
\hline Einzelhandel & 55 & 36 \\
\hline Verkehr u. Lagerei & 44 & 24 \\
\hline Information u. Kommunikation & 45 & 5 \\
\hline Finanz- und Versicherungsdienstleistungen & 48 & 23 \\
\hline Gastgewerbe u. Sonst. Dienstleistungen (DL) & 34 & 10 \\
\hline Gesundheit u. Erziehung/Unterricht & 32 & 24 \\
\hline Wirtschaftl., wissenschaftl. u. freiberufl. DL & 32 & 9 \\
\hline Gesamt & 39 & 28 \\
\hline
\end{tabular}

\begin{tabular}{|c|c|}
\hline $\begin{array}{c}\text { Anteil der begünstig- } \\
\text { ten Beschäftigten in } \\
\text { den betreffenden } \\
\text { Betrieben }\end{array}$ \\
\hline West & Ost \\
\hline 63 & 57 \\
\hline 62 & 62 \\
\hline 65 & 60 \\
\hline 66 & 55 \\
\hline 66 & 60 \\
\hline 60 & 83 \\
\hline 51 & 33 \\
\hline 54 & 84 \\
\hline 60 & 75 \\
\hline 63 & 30 \\
\hline 67 & 50 \\
\hline 64 & 59 \\
\hline
\end{tabular}

\begin{tabular}{|c|c|}
\hline $\begin{array}{c}\text { Höhe der übertarifli- } \\
\text { chen Entlohnung } \\
\text { für begünstigte } \\
\text { Beschäftigte }\end{array}$ \\
\hline West & Ost \\
\hline 18 & 12 \\
\hline 10 & 10 \\
\hline 10 & 11 \\
\hline 9 & 12 \\
\hline 10 & 12 \\
\hline 11 & 15 \\
\hline 7 & 9 \\
\hline 11 & 15 \\
\hline 12 & 14 \\
\hline 11 & 10 \\
\hline 12 & 10 \\
\hline 10 & 11 \\
\hline
\end{tabular}

* Basis: privatwirtschaftliche Betriebe ohne Landwirtschaft und Organisationen ohne Erwerbszweck.

Quelle: IAB-Betriebspanel (2013).

für den Osten seit 1996 dargestellt. Für den Anteil der Betriebe mit gesetzlich legitimierter Interessenvertretung bestätigt sich das bisherige Bild. Es lässt sich auch weiterhin weder in den alten noch den neuen Bundesländern ein eindeutiger Trend ausmachen. Die Anteilswerte bewegen sich seit einigen Jahren in beiden Landesteilen zwischen $9 \%$ und $10 \%$. In Westdeutschland liegt dies zwar um drei Prozentpunkte unterhalb des Wertes von 1996, aber z. B. nur knapp unter dem von 1993. In Ostdeutschland ist eine ähnliche Beharrlichkeit mit geringer Schwankungsbreite zu beobachten.

Mehr Bewegung - und zwar eine rückläufige - zeigt sich mit Blick auf die Beschäftigtenzahlen in den Betrieben mit Betriebsrat. $\mathrm{Zu}$ erkennen sind die zwar von Jahr zu Jahr geringen Veränderungen, wobei sich immer wieder Phasen einer (nominellen) Stabilisierung mit solchen geringer Rückgänge abwechseln. In der Gesamtschau ist aber ein klarer Abwärtstrend unverkennbar - auch unter Berücksichtigung der veränderten Fragestellung im IABBetriebspanel seit 2003, durch die die Anteilswerte um ca. einen Prozentpunkt nach unten korrigiert wurden (vgl. Ellguth/Kohaut 2005, S. 401). In der aktuellen Befragungswelle bestätigt sich für Westdeutschland der letztjährige Tiefstwert, in Ostdeutschland ergibt sich der geringste Wert seit Bestehen des IAB-Betriebspanels.
Interessantes fördert nun eine nach Betriebsgröße differenzierte Betrachtung zutage. In Abbildung 2 ist dazu die Entwicklung für die Betriebe unterschiedlicher Größenklassen dargestellt. Bei der Reform des Betriebsverfassungsgesetzes 2001 ging es dem Gesetzgeber ja unter anderem darum, die Erosionsprozesse der betrieblichen Mitbestimmung zu stoppen und durch eine Entbürokratisierung des Wahlrechts die Neugründung von Betriebsratsgremien in Kleinbetrieben zu forcieren. Unabhängig davon, ob die damals zugrunde liegende Einschätzung einer beständig erodierenden institutionellen Basis, die (ein) Ausgangspunkt für das gesetzgeberische Handeln war, den Tatsachen entsprach, wurde auch in der Folge die quantitative Entwicklung der betrieblichen Mitbestimmung vor allem mit Blick auf die Kleinbetriebe betrachtet. Nach allen vorliegenden Analysen haben sich allerdings die Erwartungen eines entsprechenden Gründungsbooms von Betriebsratsgremien nicht erfüllt (vgl. Bellmann/Ellguth 2006), wie sich auch mit unserer Zeitreihe für die Betriebe bis 50 Beschäftigte

Anzahl der Kleinstbetriebe nur ein relativ kleinerTeil der Gesamtbeschäftigten arbeitet, ergibt sich ein Anteil an Arbeitnehmern, die qua Gesetz ohne einen Betriebsrat bleiben, von $6 \%$ im Westen und $9 \%$ im Osten. 
TABELLE 6

Anteil der Betriebe bzw. Beschäftigten mit Betriebsrat 1993-2013*

Anteile in Prozent

\begin{tabular}{|c|c|c|c|c|}
\hline & $\begin{array}{r}\text { Anteil de } \\
\mathrm{Be}\end{array}$ & $\begin{array}{l}\text { iebe mit } \\
\text { rat }\end{array}$ & $\begin{array}{l}\text { Anteil der } \\
\text { Betrieber }\end{array}$ & $\begin{array}{l}\text { ftigten in } \\
\text { triebsrat }\end{array}$ \\
\hline & $\begin{array}{c}\text { West- } \\
\text { deutschland }\end{array}$ & $\begin{array}{c}\text { Ost- } \\
\text { deutschland }\end{array}$ & $\begin{array}{c}\text { West- } \\
\text { deutschland }\end{array}$ & $\begin{array}{c}\text { Ost- } \\
\text { deutschland }\end{array}$ \\
\hline 1993 & 10 & & 51 & \\
\hline 1996 & 12 & 11 & 51 & 43 \\
\hline 1998 & 10 & 9 & 50 & 40 \\
\hline 2000 & 12 & 12 & 50 & 41 \\
\hline 2001 & 12 & 12 & 50 & 41 \\
\hline 2002 & 11 & 11 & 50 & 42 \\
\hline $2003^{* *}$ & 11 & 11 & 48 & 40 \\
\hline 2004 & 10 & 9 & 47 & 40 \\
\hline 2005 & 11 & 10 & 47 & 40 \\
\hline 2006 & 10 & 10 & 46 & 39 \\
\hline 2007 & 10 & 10 & 46 & 39 \\
\hline 2008 & 10 & 9 & 45 & 37 \\
\hline 2009 & 10 & 10 & 45 & 38 \\
\hline 2010 & 10 & 10 & 45 & 37 \\
\hline 2011 & 10 & 9 & 44 & 36 \\
\hline 2012 & 9 & 9 & 43 & 36 \\
\hline 2013 & 9 & 10 & 43 & 35 \\
\hline
\end{tabular}

* Basis: privatwirtschaftliche Betriebe ab 5 Beschäftigte, ohne Landwirtschaft

und Organisationen ohne Erwerbszweck.

** Im Vergleich zum Vorjahr veränderte Fragestellung.

Quelle: IAB-Betriebspanel.
Tabelle 7 beinhaltet auch die Zahlen für Betriebe ab 21 und ab 51 Beschäftigte, um sich ein Bild von der Reichweite der betrieblichen Mitbestimmung ohne die große Masse der klein(er)en Betriebe machen zu können. Vielfach wird argumentiert, dass der geringe Deckungsgrad im kleinbetrieblichen Segment nicht verwunderlich sei, da dort andere (direkte) Formen der Partizipation möglich sind. Die Wahl eines Betriebsrats (Obmanns) wäre hier nur erforderlich, wenn diese unmittelbaren, nicht institutionalisierten (Austausch)Beziehungen nicht (mehr) funktionieren würden. Wie dem auch sei, die Ergebnisse für diese eingeschränkten Größensegmente zeigen ein deutlich positiveres Bild. Bei den Betrieben mit mehr als 50 Beschäftigten verfügt ungefähr die Hälfte über einen Betriebsrat und $70 \%$ der Beschäftigten in West- und $60 \%$ in Ostdeutschland arbeiten in diesen Betrieben. Allerdings müssen auch für diese eingeschränkten Größensegmente in unserem Betrachtungszeitraum deutliche Verluste im Deckungsgrad konstatiert werden. Wieder auf Beschäftigte bezogen sind das 9 Prozentpunkte im Westen und 13 Prozentpunkte im Osten.

Eine differenzierte Betrachtung der Verbreitung eines Betriebsrats nach der Betriebsgröße am aktuellen Rand zeigt auch genauer, dass die insgesamt nach wie vor vorhandenen West-Ost-Unterschiede auf die geringere Reichweite der betrieblichen Mitbestimmung im Segment zwischen 101 und 500 Beschäftigte in Ostdeutschland zurückzuführen sind (Tabelle 7).

Ansonsten zeigen sich wiederum die aus den Vorjahren bekannten größenspezifischen Unterschiede. Nur knapp jeder 17. Kleinbetrieb (bis 50 Beschäftigte) verfügt über einen Betriebsrat. Ab 200 Beschäftigte (Freistellungsgrenze) sind es dann $80 \%$ im Westen und $70 \%$ im Osten. Prägt in betrieblicher Perspektive die große Zahl der Kleinbetriebe das Gesamtbild, so relativiert sich deren Gewicht natürlich bei Betrachtung des auf Beschäftigte bezogenen Deckungsgrads.

Starke Unterschiede zeigen sich auch im Deckungsgrad in einzelnen Branchen, und zwar sowohl in Betriebs- als auch Beschäftigtenperspektive (Tabelle 8). Die größte quantitative Reichweite der betrieblichen Mitbestimmung besteht im traditionell stark mitbestimmten Bereich Energieund Wasserversorgung/Abfallwirtschaft/Bergbau, in den Finanz- und Versicherungsdienstleistungen, im Verarbeitenden Gewerbe und im Bereich Verkehr/Lagerei. Das untere Ende bilden die vorwiegend kleinbetrieblich strukturierten Branchen des sonstigen Dienstleitungsbereichs (inkl Gastgewerbe) und der Bauwirtschaft. Branchenspezifische Entwicklungen lassen sich leider wegen der 2009 erfolgten Umstellung auf eine neue Wirtschaftszweigsystematik (WZ 2008) nur für einen relativ kurzen Zeitraum verfolgen. Klar identifizierbar sind für die vergangenen vier Jahre Rückgänge in den Finanz- und Versicherungsdienstleistungen (in beiden Landesteilen) und im Bereich Verkehr/Lagerei in Ostdeutschland, also in Branchen, die sich bislang eher am oberen Ende der Verteilung befunden haben. 
5. Das Zusammenspiel von betrieb-

\section{licher und überbetrieblicher Interessenvertretung}

Auf das eigentümliche Ineinandergreifen der beiden formal unabhängigen Institutionen des bundesdeutschen Systems industrieller Beziehungen - Tarifautonomie und betriebliche Mitbestimmung - mit ihren wechselseitigen Abhängigkeiten und der daraus resultierenden Fähigkeit zur kooperativen Konfliktverarbeitung wurde immer wieder hingewiesen (z. B. Schmidt/Trinczek 1991; Müller-Jentsch 2003). Das Verhältnis von tarifvertraglicher und betrieblicher Regulierung bleibt auch angesichts der immer wieder nicht nur zu Wahlkampfzeiten aufflammenden politischen Diskussion um die Zukunft des dualen Systems der Interessenvertretung virulent.

Im abschließenden gemeinsamen Blick auf die betriebliche und sektorale Ebene der (gesetzlich legitimierten) Interessenvertretung soll vor allem auch ein Eindruck von den Verschiebungen im dualen System der Interessenvertretung gegeben werden. Damit gilt im Folgenden das Augenmerk vor allem der Entwicklung der sogenannten betrieblichen Vertretungslücken, also Betrieben, die zwar tarifgebunden sind, aber keinen Betriebsrat haben, und den sogenannten weißen Flecken in der Tarif- und Mitbestimmungslandschaft, wo es keinerlei gesetzlich legitimierte Interessenvertretung gibt. Die präsentierten Zahlen beziehen sich ebenfalls auf die Betriebe der Privatwirtschaft ab fünf Beschäftigte. Eine Berücksichtigung der Kleinstbetriebe, die ja nicht zur Wahl eines Betriebsrats berechtigt sind, würde bei der gemeinsamen Betrachtung des Deckungsgrades von betrieblicher Mitbestimmung und Tarifbindung ein systematisch verzerrtes Bild liefern.
ABB. 2

\section{Beschäftigte in Betrieben mit Betriebsrat 1993 - 2013*}

Angaben in Prozent

- Ost (bis 50 Beschäftigte) West (bis 50 Beschäftigte) - Ost (51-500 Beschäftigte)

- West (51 bis 500 Beschäftigte) — Ost (501 und mehr) West (501 Besch. u. m.)

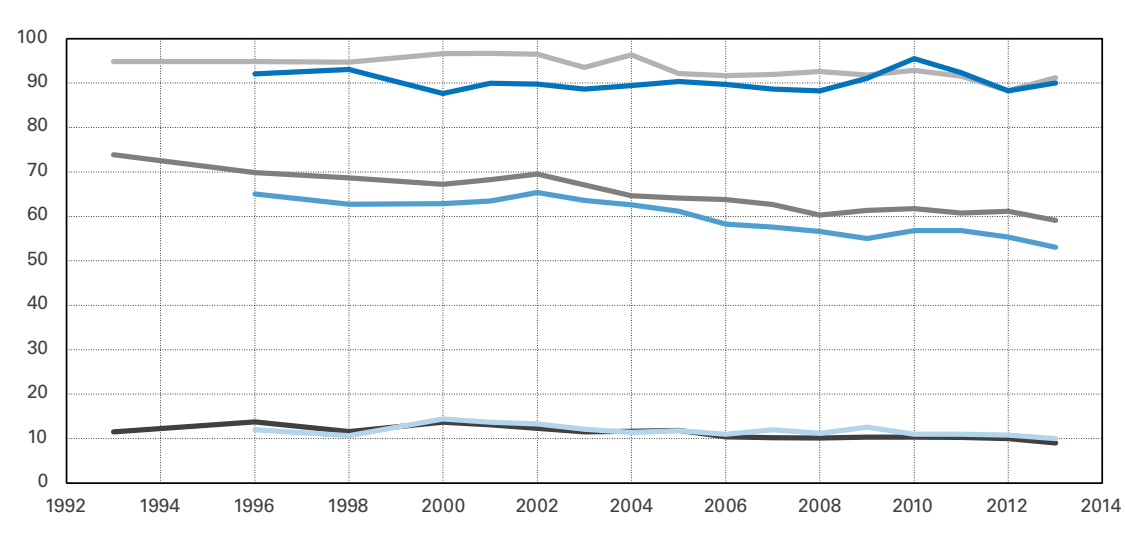

*Basis: privatwirtschaftliche Betriebe ab 5 Beschäftigte ohne Landwirtschaft und Organisationen ohne Erwerbszweck.

Quelle: IAB-Betriebspanel; Darstellung der Autoren.

Die aktuellen Zahlen bestätigen das bekannte Bild: Nur eine Minderheit der in der Privatwirtschaft beschäftigten Arbeitnehmer sind in Betrieben (ab fünf Beschäftigte) tätig, die zur Kernzone des dualen Systems der Interessenvertretung gehören. Diese Zone umfasst deutlich weniger als ein Drittel (28\%) der Beschäftigten in Westdeutschland und gerade noch ein Siebtel (15\%) in Ostdeutschland (Tabelle 9). Bemerkenswert ist die mit $10 \%$ relativ große Bedeutung von Betrieben mit Haustarifen und einem Betriebsrat

TABELLE 7

Verbreitung eines Betriebsrats nach Betriebsgröße 2013*

Anteile in Prozent

\begin{tabular}{|c|c|c|c|c|c|c|c|c|}
\hline & \multicolumn{5}{|c|}{ Betriebsgrößenklassen } & \multicolumn{3}{|c|}{ nachrichtlich: } \\
\hline & $\begin{array}{r}5-50 \\
\text { Besch. }\end{array}$ & $\begin{array}{l}51-100 \\
\text { Besch. }\end{array}$ & $\begin{array}{c}101-199 \\
\text { Besch. }\end{array}$ & $\begin{array}{c}200-500 \\
\text { Besch. }\end{array}$ & $\begin{array}{l}501 \text { u.m. } \\
\text { Besch. }\end{array}$ & $\begin{array}{c}\text { Gesamt } \\
(a b 5)\end{array}$ & $\begin{array}{r}\text { ab } 21 \\
\text { Besc }\end{array}$ & $\begin{array}{l}\text { ab } 51 \\
\text { ftigte }\end{array}$ \\
\hline \multicolumn{9}{|c|}{ Westdeutschland } \\
\hline Betriebe mit BR & 5 & 37 & 61 & 73 & 87 & 9 & 29 & 51 \\
\hline Beschäftigte mit BR & 9 & 38 & 62 & 74 & 91 & 43 & 58 & 70 \\
\hline \multicolumn{9}{|c|}{ Ostdeutschland } \\
\hline Betriebe mit BR & 6 & 35 & 53 & 68 & 89 & 10 & 26 & 45 \\
\hline Beschäftigte mit $B R$ & 10 & 36 & 54 & 69 & 90 & 35 & 48 & 60 \\
\hline
\end{tabular}

* Basis: privatwirtschaftliche Betriebe ab 5 Beschäftigte, ohne Landwirtschaft und Organisationen ohne Erwerbszweck.

Quelle: IAB-Betriebspanel (2013).

(2) 
TABELLE 8

Verbreitung eines Betriebsrats nach Branche 2013*

Anteile in Prozent

\begin{tabular}{|c|c|c|c|c|c|c|c|c|c|c|c|}
\hline & \multicolumn{10}{|c|}{ Wirtschaftszweige } & \multirow[b]{2}{*}{$\begin{array}{c}\text { Insgesamt } \\
\text { (ab 5 Besch.) }\end{array}$} \\
\hline & $\begin{array}{c}\text { Energie/ } \\
\text { Wasser/ } \\
\text { Abfall } \\
\text { Bergbau }\end{array}$ & $\begin{array}{c}\text { Verarb. } \\
\text { Gewerbe }\end{array}$ & $\begin{array}{c}\text { Bau- } \\
\text { gewerbe }\end{array}$ & Handel & $\begin{array}{l}\text { Verkehr/ } \\
\text { Lagerei }\end{array}$ & $\begin{array}{l}\text { Informat./ } \\
\text { Kommunikat. }\end{array}$ & $\begin{array}{c}\text { Finanz-I } \\
\text { Versich.-DL }\end{array}$ & $\begin{array}{l}\text { Gastgew. } \\
\text { sonst. DL }\end{array}$ & $\begin{array}{l}\text { Gesundheit } \\
\text { Erziehung/ } \\
\text { Unterricht }\end{array}$ & $\begin{array}{l}\text { Wirtschaftl. } \\
\text { wissenschaftl. } \\
\text { freiberufl. DL }\end{array}$ & \\
\hline & \multicolumn{10}{|c|}{ Westdeutschland } & \\
\hline Betriebe mit BR & 42 & 16 & 3 & 9 & 12 & 17 & 27 & 3 & 11 & 7 & 9 \\
\hline \multirow[t]{2}{*}{ Beschäftigte mit BR } & 83 & 69 & 16 & 29 & 49 & 50 & 70 & 14 & 44 & 27 & 43 \\
\hline & \multicolumn{10}{|c|}{ Ostdeutschland } & \\
\hline Betriebe mit BR & 23 & 11 & 3 & 8 & 13 & 6 & 19 & 8 & 14 & 10 & 10 \\
\hline Beschäftigte mit BR & 66 & 48 & 19 & 23 & 33 & 29 & 62 & 17 & 51 & 29 & 35 \\
\hline
\end{tabular}

*Basis: privatwirtschaftliche Betriebe ab 5 Beschäftigte, ohne Landwirtschaft und Organisationen ohne Erwerbszweck.

Quelle: IAB-Betriebspanel (2013).

TABELLE 9

\section{Tarifbindung und Betriebsrat 2013*}

Angaben des Anteils der Beschäftigten in Prozent

\begin{tabular}{|c|c|c|c|c|}
\hline & \multicolumn{4}{|c|}{ Westdeutschland } \\
\hline & \multirow{2}{*}{$\begin{array}{l}\text { Privat- } \\
\text { wirtschaft }\end{array}$} & \multicolumn{3}{|l|}{ darunter: } \\
\hline & & $\begin{array}{l}\text { Verarb. } \\
\text { Gewerbe }\end{array}$ & $\begin{array}{l}\text { Bauwirt- } \\
\text { schaft }\end{array}$ & $\begin{array}{c}\text { Dienst- } \\
\text { leistungen }\end{array}$ \\
\hline BR und Branchentarif & 28 & 47 & 14 & 22 \\
\hline BR und Haustarif & 7 & 12 & 2 & 5 \\
\hline BR und kein Tarif & 8 & 10 & 1 & 7 \\
\hline Branchentarif und kein BR & 20 & 8 & 59 & 25 \\
\hline Haustarif und kein BR & 1 & 0 & 1 & 1 \\
\hline kein Tarif und kein BR & 35 & 22 & 24 & 41 \\
\hline Gesamt & 100 & 100 & 100 & 100 \\
\hline $\begin{array}{l}\text { Beschäftigte in Betrieben } \\
\text { mit Branchentarifvertrag }\end{array}$ & 49 & 56 & 73 & 46 \\
\hline $\begin{array}{l}\text { Beschäftigte in Betrieben } \\
\text { mit Betriebsrat }\end{array}$ & 43 & 69 & 16 & 33 \\
\hline
\end{tabular}

\begin{tabular}{c|c|c|c}
\hline \multicolumn{4}{c}{ Ostdeutschland } \\
\hline $\begin{array}{c}\text { Privat- } \\
\text { wirtschaft }\end{array}$ & darunter: & \\
\cline { 2 - 4 } & $\begin{array}{c}\text { Verarb. } \\
\text { Gewerbe }\end{array}$ & $\begin{array}{c}\text { Bauwirt- } \\
\text { schaft }\end{array}$ & $\begin{array}{c}\text { Dienst- } \\
\text { leistungen }\end{array}$ \\
\hline 15 & 16 & 10 & 15 \\
\hline 10 & 12 & 5 & 11 \\
\hline 10 & 20 & 4 & 10 \\
\hline 15 & 4 & 44 & 19 \\
\hline 3 & 2 & 2 & 4 \\
\hline 16 & 46 & 36 & 41 \\
\hline 30 & 100 & 100 & 100 \\
\hline 35 & 21 & 53 & 34 \\
\hline
\end{tabular}

*Basis: privatwirtschaftliche Betriebe mit mindestens 5 Beschäftigten ohne Landwirtschaft und Organisationen ohne Erwerbszweck.

Quelle: IAB-Betriebspanel (2013); (Abweichungen von $100 \%$ aufgrund von Rundungsfehlern möglich).

in den neuen Bundesländern. Von einer betrieblichen Vertretungslücke (Betrieb ist an einen Branchentarif gebunden, hat aber keinen Betriebsrat) sind $20 \%$ der Beschäftigten in Westdeutschland, aber nur $15 \%$ in Ostdeutschland betroffen. Diese vermeintlich günstigere Situation in den neuen Bundesländern ist allerdings nur der dort geringeren Reich- weite der Tarifbindung geschuldet, die sich in deutlicher ausgeprägten „weißen Flecken der Tarif- und Mitbestimmungslandschaft“ äußert. Während in Westdeutschland „nur“ 35 \% der Beschäftigten ohne Branchentarif und ohne Betriebsrat auskommen müssen, sind dies in Ostdeutschland $46 \%$. 
Um das Bild etwas zu erweitern und mögliche branchenspezifische Unterschiede hinsichtlich der beschriebenen „Problemzonen“ zu identifizieren, sind die Ergebnisse zur Reichweite von Tarifverträgen und betrieblicher Mitbestimmung in Tabelle 9 zusätzlich für das Verarbeitende Gewerbe, die Bauwirtschaft und den Dienstleistungsbereich ${ }^{3}$ dargestellt. Für Westdeutschland lässt sich feststellen, dass die Kernzone des dualen Systems im Verarbeitenden Gewerbe knapp die Hälfte der Beschäftigten (47 \%) umfasst und damit weit über den Durchschnitt der Privatwirtschaft hinausreicht. Mit nur jedem siebten bzw. gut fünften Arbeitnehmer fallen dagegen insbesondere die Bauwirtschaft und auch der Dienstleistungsbereich deutlich ab. Im Baugewerbe haben vor allem die betrieblichen Vertretungslücken (59\%) einen ganz beträchtlichen Umfang, was wohl in erster Linie den dort geltenden Allgemeinverbindlichregelungen geschuldet ist. Der Dienstleistungsbereich tut sich hingegen mit ausgedehnten „weißen Flecken“ (41 \%) hervor. Im Verarbeitenden Gewerbe bleiben diese „Problemzonen" dagegen in relativ engen Grenzen.

In Ostdeutschland ergibt sich ein etwas anderes Bild. Hier unterscheidet sich das Verarbeitende Gewerbe weit weniger von der restlichen Privatwirtschaft, als das in den westlichen Bundesländern der Fall ist. Die Kernzone der Interessenvertretung ist mit 16 \% kaum größer als im Dienstleistungsbereich (15\%) und fällt im Vergleich zum Verarbeitenden Gewerbe Westdeutschlands (47 \%) stark ab. Offensichtlich ist in Ostdeutschland der industrielle Kernbereich nicht im gleichen überdurchschnittlichen Maße mit den beiden Institutionen der Interessenvertretung ausgestattet, wie das traditionell im Westen der Fall ist. Für den Dienstleistungsbereich ergibt sich hingegen in den neuen Bundesländern ein in Relation weit weniger negatives Bild als in Westdeutschland.

\section{LITERATUR}

Bellmann, L./Ellguth, P. (2006): Verbreitung von Betriebsräten und ihr Einfluss auf die betriebliche Weiterbildung, in: Jahrbücher für Nationalökonomie und Statistik 226 (5), S. 487-504

Ellguth, P. (2007): Betriebliche und überbetriebliche Interessenvertretung Ergebnisse aus dem IAB-Betriebspanel 2005, in: WSI-Mitteilungen 60 (3), S. 155-157, http://www.boeckler.de/wsimit_2007_03_ellguth.pdf

Ellguth, P./Kohaut, S. (2005): Tarifbindung und betriebliche Interessenvertretung: Aktuelle Ergebnisse aus dem IAB-Betriebspanels, in: WSI-Mitteilungen 58 (7), S. 398-403, http://www.boeckler.de/wsimit_2005_07_ellguth.pdf Ellguth, P./Kohaut, S. (2011): Tarifbindung und betriebliche Interessenvertretung: Aktuelle Ergebnisse aus dem IAB-Betriebspanel 2010, in: WSI-Mitteilungen 64 (5), S. 242-247, http://www.boeckler.de/wsimit_2011_05_ellguth.pdf Ellguth, P./Kohaut, S. (2012): Tarifbindung und betriebliche Interessensvertretung: Aktuelle Ergebnisse aus dem IAB-Betriebspanel 2011, in: WSI-Mitteilungen 65 (4), S. 297-305, http://www.boeckler.de/wsimit_2012_04_ellguth.pdf Ellguth, P./Kohaut, S./Möller, I. (2014): The IAB-Establishment Panel - methodological essentials and data quality, in: Journal for Labour Market Research $47(1-2)$, S. $27-41$

Müller-Jentsch, W. (2003): Re-assessing Co-determination, in: Müller-Jentsch, W./Weitbrecht, H. (Hrsg.): The changing contours of German industrial relations, München/Mering, S. 39-56

Schmidt, R./Trinczek, R. (1991): Duales System: Tarifliche und betriebliche Interessenvertretung, in: Müller-Jentsch, W. (Hrsg.): Konfliktpartnerschaft. Akteure und Institutionen der industriellen Beziehungen, München/Mering, S. 167-199

\section{AUTOREN}

PETER ELLGUTH ist Wissenschaftlicher Mitarbeiter im Institut für Arbeitsmarkt- und Berufsforschung (IAB) der Bundesagentur für Arbeit im Forschungsbereich „Betriebe und Beschäftigung". Arbeitsschwerpunkte: Arbeitsbeziehungen und betriebliche Arbeitszeitpolitik.

peter.ellguth@iab.de

SUSANNE KOHAUT, Dr., ist Wissenschaftliche Mitarbeiterin im IAB im Forschungsbereich „Betriebe und Beschäftigung". Arbeitsschwerpunkte: Arbeitsbeziehungen und Betriebsgründungen.

susanne.kohaut@iab.de
3 Der Dienstleistungsbereich in Tabelle 9 umfasst die Finanz- und Versicherungsdienstleistungen, das Gastgewerbe, die Sonstigen Dienstleistungen, das Gesundheitswesen, den Bereich Erziehung und Unterricht und die wirtschaftlichen, wissenschaftlichen und freiberuflichen Dienstleistungen. 